\title{
Imposed registries within the European postmarketing surveillance system: Extended analysis and lessons learned for regulators
}

\author{
Alexandra Pacurariu $^{1,2}$ (D) | Kelly Plueschke ${ }^{1}$ | Carla Alonso Olmo ${ }^{1}$ | Xavier Kurz ${ }^{1}$ (D)
}

${ }^{1}$ Surveillance \& Epidemiology Service, European Medicines Agency (EMA), London, UK

${ }^{2}$ Medical Informatics Department, Erasmus Medical Centre, Rotterdam, The Netherlands

Correspondence

A. Pacurariu, Surveillance \& Epidemiology Service, European Medicines Agency (EMA), London E14 5EU, UK.

Email: alexandra.pacurariu@ema.europa.eu

\begin{abstract}
Purpose: Building on previous research, we examined whether delayed study start and low patient accrual rates found in 31 postauthorization registry-based studies requested by European Medicines Agency (EMA) are maintained after 2 additional years of follow-up.

Method: The registries identified in the previous EMA study and the same methodology were used. The follow-up was extended from June 2015 to November 2017. The information available for the following variables was updated: marketing authorization status, study and registry status, study end date, planned duration, number of patients planned to be enrolled, and actual patients enrolled. Data were collected from several nonpublic in-house sources such as the study protocols, interim and final study reports, risk management plans, and periodic safety update reports.
\end{abstract}

Results: As of November 2017, 10 (32.2\%) studies were finalized (vs. 9.7\% as of June 2015), 14 (45.2\%) were still ongoing (vs. 64.5\%). Four of the ongoing studies had patients' accrual lower than $50 \%$. Six of the finalized studies had a delayed completion, with a median delay of 3 years. As of November 2017, the median patients' accrual percentages were $24 \%$ for ongoing studies (vs. $8.5 \%$ ) and $101 \%$ for finalized studies (vs. 24\%).

Conclusion: Overall, the rate of recruitment and timely finalization were improved after 2 years of additional follow-up but show that further work is needed to facilitate use of registry data for regulatory purposes, a work that has started via the EMA registry initiative.

\section{1 | BACKGROUND AND PREVIOUS FINDINGS}

Postauthorization studies based on patient registries are frequently requested to marketing authorization holders by regulatory authorities to support the benefit-risk monitoring of their licensed drugs. ${ }^{1-3} \mathrm{~A}$ previous European Medicines Agency (EMA) study examined the characteristics of registries imposed by EMA as a legally binding condition of the marketing authorization. ${ }^{1}$ Of a total of 392 products that received a positive opinion for a marketing authorization from the EMA Committee for Human Medicinal Products between January 1,
2005 and December 31, 2013, 31 registry-based studies had been imposed for 30 products (7.6\%). By June 2015, approximately $25 \%$ of these imposed studies had not started, $50 \%$ were ongoing, and less than $10 \%$ had been completed. The issues most commonly identified by the EMA in this review were delayed time to start and low patient accrual rates. Based on these results, the EMA recommended the use of existing patient registries to improve the timeliness of data collection in the postmarketing setting.

The current study examines whether these critical issues still apply to the 31 registry-based studies after a longer observation period (follow-up extended by 2 years until November 30, 2017). 
The new findings will be further considered in the EMA initiative for patient registries that aim to make better use of existing registries for regulatory purposes. ${ }^{4}$

\section{2 | METHODS}

The list of registries identified in the previous EMA study and the same methodology were used in this study. The information available for the following variables was updated: marketing authorization status, study and registry status, study end date, planned duration and number of patients planned to be enrolled, and actual patients enrolled. These data were collected from several combined nonpublic in-house sources such as study protocols, interim and final study reports, risk management plans, and periodic safety update reports.

As discussed, in the initial study, the terms "registry" and "study" are sometimes used interchangeably. In this follow-up study, we distinguished between the situation in which regulators specifically imposed a requirement to establish a registry as a systematic data collection tool and the situation in which a specific postauthorization study was imposed with specific objectives and a study design involving the use of a registry as the data collection tool. ${ }^{4}$

To illustrate the progress of the studies, we defined the "patient accrual percentage" as the number of patients currently enrolled in the study divided by the number of planned patients, and the "study progression status" as actual study duration (from first patient enrolled until last date of data collection) divided by the planned study duration.

\section{3 | RESULTS}

\section{1 | Study status}

As of November 2017, 6 studies (19.4\%) had not yet started recruiting patients (as compared with 7 as of June 2015) and 10 studies (32.2\%)

TABLE 1 Study and registry statuses over time $(n=31)$

\begin{tabular}{|c|c|c|c|c|}
\hline & \multicolumn{2}{|l|}{ Registry Status } & \multicolumn{2}{|l|}{ Study Status } \\
\hline & January 2005-June $2015^{a}$ & $\begin{array}{l}\text { January 2005-November } \\
2017\end{array}$ & $\begin{array}{l}\text { January 2005-June } \\
2015\end{array}$ & $\begin{array}{l}\text { January 2005-November } \\
2017\end{array}$ \\
\hline & $\mathrm{N}(\%)$ & $\mathrm{N}(\%)$ & $\mathrm{N}(\%)$ & $\mathrm{N}(\%)$ \\
\hline Not started ${ }^{b}$ & $7(22.6 \%)$ & $5(16.1 \%)$ & $7(22.6 \%)$ & $6(19.4 \%)$ \\
\hline Ongoing & $20(64.5 \%)$ & $10(32.3 \%)$ & $20(64.5 \%)$ & $14(45.2 \%)$ \\
\hline Finalized & $3(9.7 \%)$ & $10(32.3 \%)$ & $3(9.7 \%)$ & $10(32.2 \%)$ \\
\hline Missing information ${ }^{c}$ & $1(3.2 \%)$ & $1(3.2 \%)$ & $1(3.2 \%)$ & $1(3.2 \%)$ \\
\hline Open ended ${ }^{d}$ & $\mathrm{~N} / \mathrm{A}$ & $5(16.1 \%)$ & $\mathrm{N} / \mathrm{A}$ & $\mathrm{N} / \mathrm{A}$ \\
\hline
\end{tabular}

${ }^{a}$ For the period between January 2005 and June 2015, the registry and study status are identical, as the first European Medicines Agency study did not differentiate between these 2 terms.

${ }^{\text {b}}$ All the studies/registries in the "not started" category are related to products for which the marketing authorizations have been withdrawn.

'Missing information about the end date/study status.

${ }^{\mathrm{d}}$ The registries are run independently from the conduct of the studies.
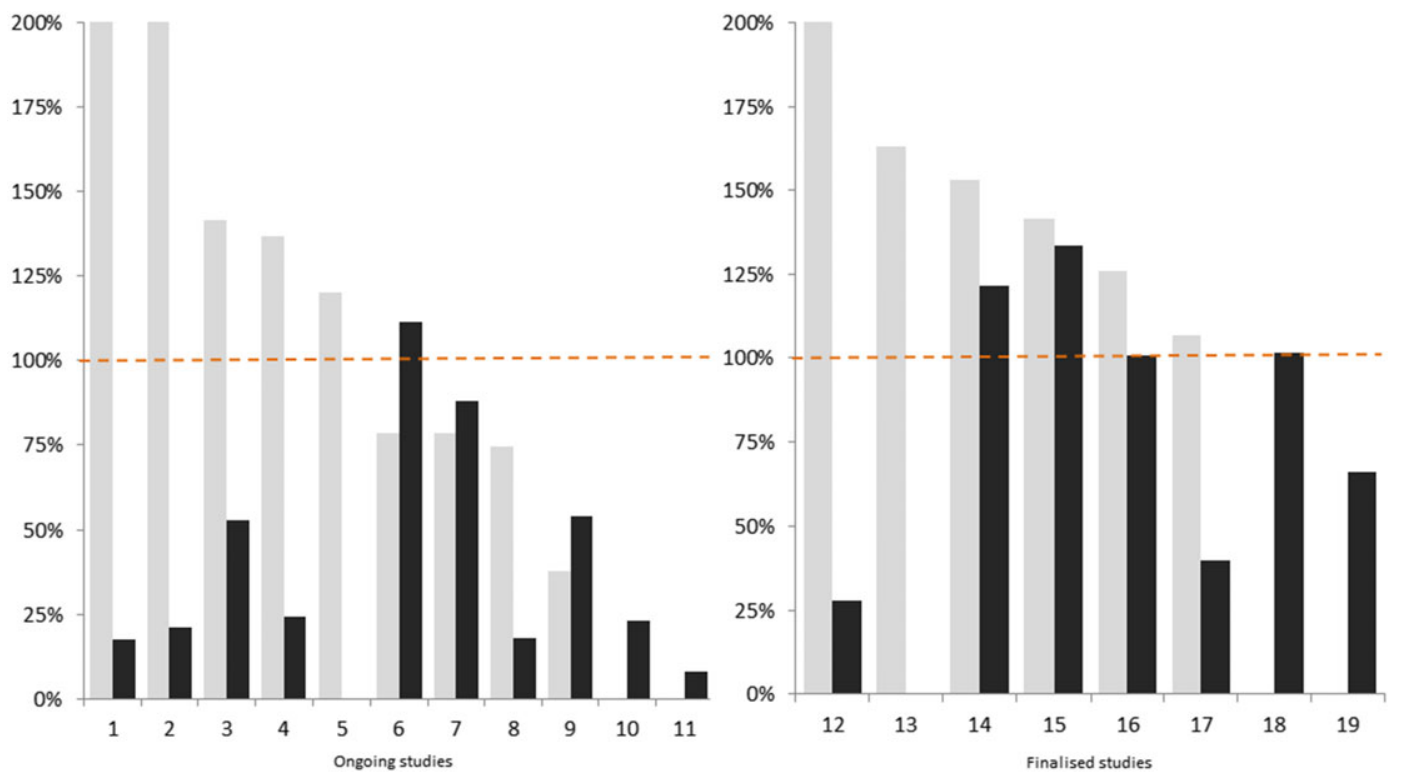

FIGURE 1 Accrual percentage in relation to study progression status. Each number on $x$-axis represents a different study and is described by 2 bars: The light gray bar represents the study progression status, and the black bar represents patient accrual percentage. Studies with "progression" above $100 \%$ are running longer than planned [Colour figure can be viewed at wileyonlinelibrary.com] 
had been finalized (as compared with 3 as of June 2015). Fourteen studies were still ongoing (45.2\%), compared with 20 (64.5\%) in the previous period (see Table 1).

For 11 of the 14 ongoing studies and for 8 of the 10 finalized studies, available information allowed to calculate either study progression status or accrual percentage (Figure 1). Among the ongoing studies, 4 were still within the original agreed timeframe (progression status $<100 \%$ ) with 1 study already surpassing the targeted number of patients, and 5 were delayed by a median of 5 years (range: 17 years) with patients' accrual lower than $50 \%$ for 4 of them (1 had missing data). Among the finalized studies, 6 had a delayed completion with a median delay of 3 years (range: 2-6 years). Three finalized studies managed to reach or surpass $100 \%$ of patients' accrual.

Fifty percent of studies conducted within disease registries and $33 \%$ of studies conducted within product registries were delayed compared with the planned finalization date.

As of November 2017, the median patients' accrual percentages were $24 \%$ for ongoing studies and $101 \%$ for finalized studies, as compared with $8.5 \%$ and $24 \%$ in June 2015.

\section{2 | Registry status}

Compared with the previous period, 2 (6.4\%) more registries started data collection and 7 (22.6\%) more were finalized. Five registries (16\%) are open-ended (Table 1).

Both for studies and registries, missing data were common, highlighting an omission to report essential information to regulators. The following variables were most affected: planned duration $(26 \%$ missing data), planned patients ( $9.7 \%$ missing data), number of actual patients, and study end date (6.5\% missing data).

\section{4 | DISCUSSION AND CONCLUSION}

This update shows that $32.2 \%$ of registry-based studies were finalized as of November 2017 as compared with $9.7 \%$ as of June 2015. The overall patient accrual was higher than in the first period, both for ongoing and finalized studies.

Overall, the conclusions reached in the initial EMA study remain valid after a longer follow-up period: Both the rate of recruitment and timely finalization are challenging for registry-based studies. A reason may be that most of the investigated products are orphan or specialized products with a very small target population. This should be taken into consideration during the study design stage when realistic study timeframes and planned patient numbers should be proposed.

Although several nonpublic data sources were consulted, consistent information on planned duration, planned/actual patients, and study end date was still difficult to identify. The current results therefore support the need for more systematic, transparent, and standardized approach for the conduct and reporting of registry-based studies. It should be highlighted that most of the studies identified in the initial study were imposed before the new EU pharmacovigilance legislation took effect in July $2012 .{ }^{5}$ The new legislation requires the review of the study protocols and results by the Pharmacovigilance and Risk Assessment Committee and the registration of imposed postauthorization safety studies in the EU PAS Register. ${ }^{6}$ These measures should, at least in theory, lead to an improvement in reporting. An evaluation of registry-based studies imposed after July 2012 should identify whether improvements have been made following the new EU pharmacovigilance legislation, and this may be an area of future research for EMA.

Most registries used for imposed studies in this review are product registries, with patient entry based on exposure to a specific drug. The EMA's Patient Registries Initiative initiated in $2015^{4}$ strongly recommends use of "disease registries," covering a wider population defined by a particular condition that is followed over time regardless of the products administered. As disease registries are generally integrated in health systems and clinical practice, they may be associated with better accesibility to data.

European Medicines Agency is already aware of the challenges in using registries to monitor the safety of marketed medicines. To this end, EMA has held 2 specific registry workshops on cystic fibrosis and multiple sclerosis diseases. ${ }^{7,8}$ The analysis performed at these workshops highlighted that registries do not often collect routinely all the data needed for a postauthorization study. This may be due to the limited standardization of data elements captured by national and international registries, the inadequate data quality control, the time lag between the data collection and data availability, as well as a possible misunderstanding by regulators of the ability of a given registry to collect data on specific regulatory concerns. These aspects are currently being addressed through the EMA initiative for patient registries focusing on efficient collaboration among medicine regulators, pharmaceutical companies, and various registry coordinators. A certification process could ensure users that the data are of acceptable quality for regulatory purposes. As a first example in this direction, the European Cystic Fibrosis Society Patient Registry ${ }^{9}$ undertook an EMA qualification procedure to assess whether its' data collection system is adequate to support regulatory decision making. $^{10}$

Overall, the data from this report are encouraging but show that further work are needed to facilitate use of registry data for regulatory purposes, a work that has already been started via the EMA registry initiative.

\section{DISCLAIMER}

The views expressed in this article are the personal views of the authors and may not be understood or quoted as being made on behalf of or reflecting the position of the European Medicines Agency or one of its committees or working parties.

\section{ETHICS STATEMENT}

Since no human or animal subjects are involved, no ethical issues are applicable to this research.

\section{ACKNOWLEDGEMENTS}

The authors are grateful to Jane Moseley and Kevin Blake for their useful comments during the revision of this manuscript.

\section{CONFLICT OF INTEREST}

The authors have no conflict of interest to declare. 


\section{ORCID}

Alexandra Pacurariu (D) http://orcid.org/0000-0002-2217-0540

Xavier Kurz (D) http://orcid.org/0000-0002-9838-7754

\section{REFERENCES}

1. Bouvy JC, Blake K, Slattery J, De Bruin ML, Arlett P, Kurz X. Registries in European post-marketing surveillance: a retrospective analysis of centrally approved products, 2005-2013. Pharmacoepidemiol Drug Saf. 2017 Dec;26(12):1442-1450.

2. Jonker CJ, van den Berg HM, Kwa MSG, Hoes AW, Mol PGM. Registries supporting new drug applications. Pharmacoepidemiol Drug Saf. 2017 Dec;26(12):1451-1457.

3. Zhao Y, Pinnow E, Major JM. A systematic assessment of key design and performance characteristics of drug exposure registries requested by the U.S. Food and Drug Administration. Pharmacoepidemiol Drug Saf. 2018 Jan 19. https://doi.org/10.1002/pds.4386

4. EMA. Initiative for patient registries [Internet]. 2015 [cited 2018 Feb 13]. Available from: http://www.ema.europa.eu/docs/en_GB/document_library/Other/2015/10/WC500195576.pdf

5. European Commision. Commission Implementing Regulation 520/ 2012 [Internet]. [cited 2018 Feb 13]. Available from: https://ec. europa.eu/health/sites/health/files/files/eudralex/vol-1/reg_2012_ 520/reg_2012_520_en.pdf

6. The European Union electronic Register of Post-Authorisation Studies [Internet]. [cited 2018 Mar 7]. Available from: http://www.encepp.eu/ encepp_studies/indexRegister.shtml
7. European Medicines Agency. Report on multiple sclerosis registries [Internet]. 2017 [cited 2018 Feb 27]. Available from: http://www. ema.europa.eu/docs/en_GB/document_library/Report/2017/10/ WC500236644.pdf

8. European Medicines Agency. Report on cystic fibrosis registriesworkshop 1 [Internet]. 2017 [cited 2018 Feb 27]. Available from: http://www.ema.europa.eu/docs/en_GB/document_library/Report/ 2017/10/WC500236631.pdf

9. European Cystic Fibrosis Society. Qualification opinion for the European Cystic Fibrosis Patient Registry [Internet]. 2017 [cited 2018 Feb 27]. Available from: http://www.ema.europa.eu/docs/en_GB/document_library/Regulatory_and_procedural_guideline/2018/02/ WC500243542.pdf

10. European Medicines Agency. Scientific advice and protocol assistance -qualification of novel methodologies for medicine development [Internet]. [cited 2018 Feb 27]. Available from: http://www.ema. europa.eu/ema/index.jsp?curl=pages/regulation/document_listing/ document_listing_000319.jsp\%26amp\%3Bmid= WC0b01ac0580022bb0

How to cite this article: Pacurariu A, Plueschke K, Olmo CA, Kurz X. Imposed registries within the European postmarketing surveillance system: Extended analysis and lessons learned for regulators. Pharmacoepidemiol Drug Saf. 2018;1-4. https://doi. org/10.1002/pds.4449 